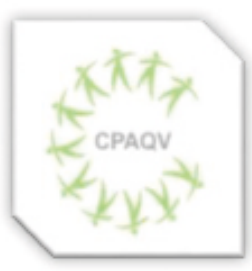

ISSN: $2178-7514$

Vol. 12 | No. 1| Ano 2020
ARTIGO ORIGINAL

\section{ESTRATÉGIAS DE FISIOTERAPIA DOMICILIAR NO TRATAMENTO DE CRIANÇAS COM PARALISIA CEREBRAL}

Hélade Beatriz Farias Figueiredo'; Ingrid Ribeiro de Ribeiro'; Thamires Ferreira Côrrea ${ }^{1}$; Thatiane Belém Rosa ${ }^{1}$; Larissa Salgado de Oliveira Rocha ${ }^{2}$; George Alberto da Silva Dias ${ }^{3}$

\section{RESUMO}

Objetivo: Analisar a influência de estratégias fisioterapêuticas, com características lúdicas, realizadas no âmbito domiciliar no tratamento de crianças com Paralisia Cerebral. Métodos: Estudo de caso com duas crianças com Paralisia Cerebral do tipo espástica na faixa etária de 5 e 10 anos que estavam em atendimento no Ambulatório de Fisioterapia Neuropediátrica. O instrumento de coleta de dados foi a escala Medida da Função Motora Grossa. As dimensões utilizadas foram a Dimensão B (sentar) e D (levantar). Foi realizada a construção e entrega de uma maleta a partir do PJBL com atividades funcionais lúdicas aos responsáveis para estes darem continuidade ao tratamento da criança em domićlio nos dias em que não houve tratamento no ambulatório. Os dados coletados foram analisados por meio do software Excel $^{\circledR} 2010$ e por análise descritiva. Resultados: O paciente W.G apresentava limitação apenas no membro superior esquerdo, durante a reavaliação após 6 sessões, fora notado que o paciente utilizava o membro superior esquerdo nesta mesma atividade. O paciente A.J. não apresentou melhora após o tratamento. Conclusão: Concluiu-se que a terapia domiciliar por meio da maleta lúdica não gerou tantos efeitos significativos, haja vista que o tempo de tratamento fora curto somada a limitações dos pesquisadores em saberem a quantidade e a qualidade dos estímulos que os responsáveis deram em casa aos participantes.

Palavras-chave: Paralisia Cerebral; Fisioterapia; Lúdico; Serviço de Assistência Domiciliar; Terapia por Exercício.

\section{ABSTRACT}

Objective: Analyze the influence of physiotherapeutic strategies, with playful characteristics, performed at home in the treatment of children with Cerebral Palsy. Methods: Case study with two children with spastic Cerebral Palsy in the age group of 5 and 10 years who were in care at the neuropediatric physiotherapy outpatient clinic. The data collection instrument was the Thick Motor Function Measurement scale. The dimensions used were Dimension B (sit) and D (lift). The construction and delivery of a briefcase from the PJBL with playful functional activities was carried out to those responsible for continuing the treatment of the child at home on the days when there was no treatment in the outpatient clinic. The collected data were analyzed using excelsoftware ${ }^{\circledR} 2010$ and descriptive analysis. Results: The W.G patient presented limitation only in the left upper limb, during the reassessment after 6 sessions, it was noticed that the patient used the left upper limb in this same activity. Patient A.J. showed no improvement after treatment. Conclusion: It was concluded that home therapy through the playful suitcase did not generate so many significant effects, since the treatment time was short added to the limitations of the researchers in knowing the quantity and quality of stimuli that the responsible people gave at home to the participants.

Keywords: Cerebral Palsy; Physiotherapy; Ludic; Home Care Services; Exercise Therapy.

Autor de correspondência

Hélade Beatriz Farias Figueiredo

DOI: doi.org/10.36692/cpaqv-v12n1-21

heladebff@gmail.com 


\section{INTRODUÇÃO}

A Paralisia Cerebral (PC) é causada por uma lesão no sistema nervoso central (SNC) que pode ocorrer durante a fase pré, peri ou pósnatal ${ }^{(1)}$. Apesar do prognóstico de aspecto não evolutivo da lesão, os sinais e sintomas podem alterar no decorrer do tempo ${ }^{(2)}$. Nesse contexto, os indivíduos com PC manifestam alterações posturais, de tônus muscular e reflexos, limitando seu desempenho funcional, principalmente no que tange a falta de controle postura ${ }^{(3)}$.

A PC pode ser classificada em três tipos: espástica, discinética e atáxica. Essa classificação se dá a partir da característica clínica predominante. A discinética ocorre por lesão no sistema extrapiramidal, majoritariamente nos núcleos da base, e é caracterizada por movimentos e posturas atípicos, como distonia e coreoatetose. A atáxica ocorre por uma disfunção no cerebelo, caracterizada por uma dissinergia que gera alterações na coordenação dos movimentos, sendo evidente na marcha. A PC espástica é causada por lesão no sistema piramidal caracterizada por hipertonia e aumento dos reflexos miotáticos, clônus e sinal de babinski ${ }^{(4)}$.

Os pacientes com PC espástica podem apresentar fraqueza nos músculos flexores plantares, extensores de quadril e joelho ${ }^{(5)}$, além de sedestação instável, o que pode trazer consequências negativas durante a realização de atos motores ${ }^{(6)}$.

Neste cenário, a Fisioterapia pode proporcionar ganhos por meio de vários recursos, entre eles o brincar, estimulando assim a criança a participar do tratamento de maneira mais assídua e com maior adesão, gerando ganhos funcionais ${ }^{(7)}$.

Contudo, sabe-se que a criança com PC possui muitas limitações motoras, diante das quais a fisioterapia realizada somente à nível ambulatorial se faz insuficiente para estimular o desenvolvimento da criança de forma eficaz ${ }^{(8)}$. Diante disso, visando maximizar o tratamento fisioterapêutico, a reabilitação em casa estende os benefícios do tratamento para além do centro de reabilitação, estimulando o melhor desempenho da criança a partir da inclusão da família no processo de reabilitação ${ }^{(8,9)}$.

\section{OBJETIVO}

Analisar a influência de estratégias fisioterapêuticas lúdicas realizadas no âmbito domiciliar no tratamento de crianças com Paralisia Cerebral.

\section{MÉTODOS}

A pesquisa teve início após aprovação do Comitê de Ética em Pesquisa do Centro de Saúde Escola do Marco (CSEM) (Parecer n 3.694.045). CAAE número: 23202419.0.0000.5174. Tratase de um estudo de caso, seguindo as normas da Resolução 466/12 do Conselho Nacional de Saúde, relativa à pesquisa com seres humanos. Assim, a pesquisa foi realizada após todos os todos os participantes assinaram o termo de consentimento livre e esclarecido e o termo de assentimento livre e esclarecido.

Participaram do estudo duas crianças diagnosticadas com paralisia cerebral do tipo 
espástica na faixa etária de 5 e 10 anos do sexo masculino, que estavam em atendimento no ambulatório de Fisioterapia Neuropediátrica vinculado a Unidade de Ensino e Assistência de Fisioterapia e Terapia Ocupacional (UEAFTO), da Universidade do Estado do Pará (UEPA), no Centro de Ciências Biológicas da Saúde (CCBS) Campus II, no período de Outubro a Novembro de 2019. Estes indivíduos foram selecionados por meio da amostragem não probabilística por conveniência.

Assim, foram excluídos do estudo pacientes que apresentaram déficit cognitivo e alterações nos sentidos especiais (cegueira e surdez) que os impediam de realizar as atividades propostas pela pesquisa.

Os pacientes com PC apresentam diversas consequências motoras, necessitando, assim, da atuação da Fisioterapia. Desta forma, utilizou-se a escala Medida da Função Motora Grossa (GMFM), a qual mensura a função motora de acordo com sua limitação funcional. As dimensões utilizadas foram a Dimensão B, referente ao sentar e a Dimensão $\mathrm{D}$, referente ao levantar.

A avaliação foi realizada pelos próprios pesquisadores, no dia e horário de atendimento do paciente no ambulatório de Fisioterapia neuropediátrica, em um ambiente reservado, visando o bem-estar do participante e fidedignidade das respostas. Durante a avaliação, houve o acompanhamento das profissionais técnicas do ambulatório. Após seis sessões domiciliares, as crianças foram novamente avaliadas a fim de verificar os efeitos funcionais da abordagem fisioterapêutica.

Foi entregue uma maleta lúdica aos responsáveis das crianças para estes darem continuidade ao tratamento em domicílio nos dias em que não houve tratamento na UEAFTO. A maleta lúdica foi desenvolvida utilizando as estratégias do método de ensino Project Based Learning (PjBL), onde foi buscado não somente a criação do produto (maleta) como também o fomento de outras habilidades transversais de caráter imprescindível para a formação atual do fisioterapeuta.

Executando a criação da maleta conforme diretrizes do PjBL, para o tratamento em domicílio, os pesquisadores a desenvolveram construída com materiais economicamente acessíveis, como papel A4, EVA, massinha de modelar, TNT, garrafas PET, espeto de madeira, cartolina, fita colorida, cola, tesoura e tinta. Ela continha jogos que estimularam o equilíbrio sentado, por meio de manutenção isométrica e isotônica do tronco e que estimularam o ortostatismo, por meio de movimentos ativos e resistidos dos membros inferiores. Os jogos utilizados foram:

- Twister de Bichinho: O objetivo do jogo era desenvolver força nos membros inferiores através de um painel, confeccionado com TNT e com imagens de animais coladas no mesmo. Para realizar a atividade, o responsável deveria escolher um animal e pedir para a criança pular em cima da imagem escolhida. Desta forma, seria trabalhada a força de membro inferior aliada ao controle de tronco e equilíbrio. 
- Pesinhos com Animais: o objetivo do jogo foi desenvolver força nos membros inferiores, especialmente no quadríceps e isquiotibiais para que o ortostatismo e a sedestação pudessem ser melhorados. Isso se deu por meio da utilização de pesos com garrafas cheias de areia com o desenho de algum animal colorido. A criança deveria estar sentada e o peso colocado em seu tornozelo. Diante disso, conforme instruções da ficha, o responsável deveria ajudar a criança a fazer a extensão do joelho. $\mathrm{Na}$ ficha estava escrito "levante o animal "X" até a altura do seu joelho". Desta forma, a criança trabalharia músculos do membro inferior importantes para o ortostatismo e a sedestação.

- Estátua: o objetivo do jogo foi desenvolver o equilíbrio em pé. O comando era dado pelo responsável que colocaria ou cantaria uma música e falaria "estátua" no momento que decidir, de forma que o paciente estivesse em uma posição que tenha que se equilibrar.

- Amarelinha dos Bichinhos: o objetivo do jogo era desenvolver o equilíbrio em pé. O responsável colocaria o tapete de bichinhos no chão e daria os comandos para a criança pular sobre os bichos apresentados no tapete.

Foi utilizado o software Excel® 2010 para entrada dos dados e confecção das tabelas. Utilizou-se análise descritiva dos dados.

\section{RESULTADOS}

Paciente W.G, sexo masculino, cinco anos, diagnosticado com paralisia cerebral do tipo espástica, apresenta como comorbidades hiperatividade e epilepsia. Durante a avaliação inicial foram encontradas algumas perdas funcionais. $\mathrm{Na}$ dimensão $\mathrm{B}$ referente ao sentar, foram encontradas alterações nos itens 27 "sentados sobre o tapete: toca o brinquedo colocado a $45^{\circ}$ atrás do lado esquerdo da criança, retorna para a posição inicial", item 29 "sentada sobre o lado esquerdo: mantém braços livres por 5 segundos" e item 32 "sentada sobre o tapete com pés para a frente: atinge 4 apoios sobre o lado esquerdo". Nos três itens citados o paciente alcançou pontuação 2 (completa parcialmente). Nos demais itens relacionados à dimensão sentar, a criança alcançou pontuação 1 (inicia).

$\mathrm{Na}$ dimensão D referente as habilidades em pé, o paciente apresentou dificuldades nos itens 57 “em pé: levanta o pé esquerdo, braços livres por 10 segundos", item 60 “ajoelhada: atinge a posição em pé passando pela posição semi ajoelhada sobre o joelho direito sem usar os braços" e item 61 "ajoelhada: atinge a posição em pé passando pela posição semi ajoelhada sobre o joelho esquerdo sem usar os braços". Nos três itens citados, o infante apresentou pontuação 2 (completa parcialmente). Além disso, demonstrou alteração também no item 58 “em pé: levanta o pé direito e braços livres por 10 segundos". Neste, o avaliado alcançou pontuação 3 (não completa).

$\mathrm{Na}$ sua reavaliação, fora notado que o protocolo de tratamento domiciliar expôs efeito, na dimensão B sentado, somente no item número 27 “sentada sobre o tapete: toca o brinquedo colocado a $45^{\circ}$ atrás do lado esquerdo da criança, retorna para a posição inicial". $\mathrm{Na}$ avaliação 
fora pontuado 2 (completa parcialmente) e na reavaliação pontuou 1 (inicia). $\mathrm{Na}$ dimensão $\mathrm{D}$ em pé, demonstrou efeito no item 59 "sentada em banco pequeno: atinge a posição em pé sem usar os braços", na avaliação pontuou 3 (não completa) e na reavaliação pontuou 2 (completa parcialmente). Ademais, mudanças também foram notadas nos itens 62 "em pé: abaixa-se com controle para sentar no chão, braços livres" e 63 “em pé: agacha-se, braços livres”, na avaliação nos dois itens pontuou 2 (completa parcialmente) e na reavaliação pontuou 3 (não completa).

Para o paciente A.J, sexo masculino, seis anos, diagnosticado com paralisia cerebral do tipo diplégica espástica, não apresentou outras comorbidades e não realiza nenhuma atividade física. Durante a avaliação inicial, foi notado que o paciente não conseguia manter-se de pé sem ajuda, por isso utilizava cadeira de rodas, o que impossibilitou de ser testado os itens 35, 36 e 37 da dimensão B (sentar) e a dimensão D (em pé) nos quais o mesmo deveria se manter em ortostatismo, marcando assim a opção "não testada".

No que tange ao restante dos itens da dimensão B, a maioria dos itens marcados foi a pontuação 1 (inicia) com exceção do item 30, na qual foi marcada a opção 2 (completa parcialmente), o item 33, no qual a criança não conseguiu iniciar o movimento (opção 0), os itens 20, 29 e 34 nos quais o mesmo não conseguiu completar o movimento e nos itens que ficaram impossibilitados de serem testados (35, 36 e 37), sendo assim marcando a opção NT (não testado).
$\mathrm{Na}$ reavaliação foi realizada após o atendimento da criança, havendo mudanças desde a avaliação inicial. A maioria dos itens marcados na dimensão $B$ ainda foram as opções 1 (inicia), porém houve mais resultados nas opções 2 (completa parcialmente) do que na avaliação inicial.

Ademais, o paciente não conseguiu iniciar o movimento do item 29 sendo marcado a pontuação 0 . O mesmo também não conseguiu completar as atividades dos itens 32, 33 e 34, pontuando 3. Por fim, não houve teste nos itens 35, 36 e 37 da dimensão B e a dimensão D inteira, nos quais foram marcados a opção NT, conforme mostra a tabela 1 .

Tabela 1 - Avaliação e reavaliação dos pacientes com paralisia cerebral espástica.

\begin{tabular}{|c|c|c|c|c|}
\hline \multirow{2}{*}{ DIMENSÄO B } & \multicolumn{2}{|c|}{ AVALIAÇÃO } & \multicolumn{2}{|c|}{ REAVALIAÇÃO } \\
\hline & $\overline{W \cdot G}$ & A. J & W. G & A. J \\
\hline 18 & 1 & 1 & 1 & 1 \\
\hline 19 & 1 & 1 & 1 & 2 \\
\hline 20 & 1 & 3 & 1 & 2 \\
\hline 21 & 1 & 1 & 1 & 1 \\
\hline 22 & 1 & 1 & 1 & 1 \\
\hline 23 & 1 & 1 & 1 & 1 \\
\hline 24 & 1 & 1 & 1 & 1 \\
\hline 25 & 1 & 1 & 1 & 1 \\
\hline 26 & 1 & 1 & 1 & 2 \\
\hline 27 & 2 & 1 & 1 & 2 \\
\hline 28 & 1 & 1 & 1 & 1 \\
\hline 29 & 2 & 3 & 2 & 0 \\
\hline 30 & 1 & 2 & 1 & 2 \\
\hline 31 & 1 & 1 & 1 & 1 \\
\hline 32 & 2 & 1 & 2 & 3 \\
\hline 33 & 1 & 0 & 1 & 3 \\
\hline 34 & 1 & 3 & 1 & 3 \\
\hline 35 & 1 & NT & 1 & NT \\
\hline 36 & 1 & NT & 1 & NT \\
\hline 37 & 1 & NT & 1 & NT \\
\hline
\end{tabular}




\begin{tabular}{lllll} 
DIMENSÄO D & & & \\
52 & 1 & NT & 1 & NT \\
53 & 1 & NT & 1 & NT \\
54 & 1 & NT & 1 & NT \\
55 & 1 & NT & 1 & NT \\
56 & 1 & NT & 1 & NT \\
57 & 2 & NT & 2 & NT \\
58 & 3 & NT & 3 & NT \\
59 & 1 & NT & 2 & NT \\
60 & 2 & NT & 2 & NT \\
61 & 2 & NT & 2 & NT \\
62 & 1 & NT & 2 & NT \\
63 & 1 & NT & 2 & NT \\
64 & 1 & NT & 1 & NT \\
\hline Não testado (NT). & & & &
\end{tabular}

\section{DISCUSSÃO}

O paciente W.G tem uma capacidade funcional boa, pratica o esporte judô, apresentando limitação apenas no lado esquerdo, mais precisamente no membro superior esquerdo. Este se apresenta em flexão e com redução de resistência muscular. Neste contexto, poucas alterações funcionais foram encontradas nele, no item 27 da dimensão sentar, na avaliação este rodava o tronco para tocar o brinquedo com o braço direito negligenciando a utilização com o braço esquerdo.

Este fato pode ser interpretado como a presença de negligência motora já que muitas crianças com Paralisia Cerebral apresentam alterações funcionais no membro superior hemiparético, a qual se assemelha a distúrbios de negligência motora. A negligência unilateral, a qual caracteriza-se pela redução do uso do membro afetado nas atividades motoras, levando ao desuso ${ }^{(14)}$. Logo, o paciente refere dificuldade em se orientar em direção a algo localizado no espaço, direcionar a atenção e iniciar a ação com o membro em questão.
Ao final de seis sessões durante a reavaliação fora notado que o paciente já utilizava o membro superior esquerdo nesta mesma atividade. Indicando que a terapia domiciliar, por meio dos exercícios de membro superior podem ter gerado um efeito positivo.

Nos itens 59, 62 e 63 da dimensão em pé, inicialmente o avaliado completou totalmente a atividade. No entanto, na reavaliação este realizou apenas parcialmente. $O$ resultado pode estar atrelado ao laço que fora criado entre a criança e os avaliadores. Visto que esta demonstrou ter desenvolvido um vínculo com estes, sendo assim, na reavaliação a criança estava mais agitada, falante e dispersa mesmo com as tentativas de manter o foco da criança pelos avaliadores.

Além disso, deve-se levar em consideração o diagnóstico de hiperatividade da criança avaliada, pode-se atrelar este resultado a dispersão. Nesse contexto, hiperatividade é manifestada pela agitação, intensa movimentação, impulsividade, desatenção, dificuldade de manter o foco e agressividade em alguns casos. Essas dificuldades são percebidas em ambientes sociais durante trabalhos organizados e concentrados, ao qual a criança recebe instruções sobre o que fazer $^{(15)}$.

Durante a avaliação inicial do voluntário A.J foi notado que o mesmo não conseguia permanecer em ortostatismo sem ajuda da mãe, da fisioterapeuta ou dos avaliadores. Nesse contexto, nos pacientes com PC, a marcha deficitária depende do grau e do tipo de envolvimento motor ${ }^{(10)}$. O desenvolvimento 
neuropsicomotor tardio, alterações na postura e de cunho musculoesqueléticos também podem interferir na manutenção do ortostatismo, porém o principal fator é a dificuldade de manter o equilíbrio necessário para a marcha ${ }^{(11)}$.

Foi percebido que a criança tinha dificuldades para realizar atividades que necessitavam de controle de tronco, uma vez que a fraqueza dos músculos do tronco, principalmente abdominais, é comum em pacientes com PC moderada ou severa ${ }^{(13)}$. Sendo a ativação dessa musculatura relevante para a manutenção da estabilidade durante atividades que envolvam os membros superiores e inferiores ${ }^{(12)}$.

$\mathrm{Na}$ reavaliação não foi identificado alterações expressivas mesmo realizando as atividades em domicilio, porém, a gravidade neuromotora influencia no desempenho funcional, mas não é determinante, visto que a mesma abrange não somente as propriedades intrínsecas da criança, mas também pelas demandas da tarefa e pelo meio que ela está inserida e interagindo ${ }^{(16)}$. O desempenho da criança vai além das atividades fisioterapêuticas realizadas no ambulatório.

A partir disso, no fim da pesquisa não se pôde obter resultados tão significativos. Haja vista que o tempo de tratamento fora curto e dificuldade dos pesquisadores em saberem a quantidade e a qualidade dos estímulos que os responsáveis deram em casa aos participantes apesar de todos os esforços para explicar a estes a melhor forma de realizar os exercícios propostos.

\section{CONCLUSÃO}

Infere-se que as crianças com paralisia cerebral apresentam redução de sua capacidade funcional, afetando sua qualidade de vida e boa execução de suas atividades de vida diária. Desta forma, expressa-se a importância de um acompanhamento fisioterapêutico a este público, visando reduzir os déficits motores. Porém, a terapia domiciliar por meio da maleta lúdica não gerou efeitos significativos.

Acredita-se que novas pesquisas precisam ser realizadas com o tema em questão, objetivando esclarecer os efeitos da terapia domiciliar lúdica com coadjuvante ao tratamento fisioterapêutico convencional.

\section{REFERÊNCIAS}

1 - Ireno JM, Chen N, Zafani MD, Baleotti LR. O uso de órteses em crianças com paralisia cerebral: percepção dos cuidadores.Cad. Bras. Ter. Ocup. 2019 [citado Out 2019]. Acesso em: http:/ / www. scielo.br/pdf/cadbto/v27n1/pt_2526-8910cadbto-27-01-00035.pdf

2 - Baxter P, Morris C, Rosenbaum P, Paneth N, Leviton A, Goldstein M, et al. The Definition and Classification of Cerebral Palsy. Dev. Med. Child Neurol. 2007 [citado Out 2019]. Acesso em: https:/ / onlinelibrary.wiley.com/doi/ pdf/10.1111/j.1469-8749.2007.00201.x

3 - Santamaria V, Rachwani J, Saavedra SL, Woollacott MH. The impact of Segmental Trunk Support on Posture and Reaching in Children With Cerebral Palsy. Pediatric Physical Therapy. 2016 [citado Out 2019]. Acesso: https://www. researchgate.net/publication/304406222_ Effect_of_Segmental_Trunk_Support_on_ Posture_and_Reaching_in_Children_With_ Cerebral_Palsy

4 - Brasil. Diretrizes de atenção à pessoa com paralisia cerebral. Ministério da Saúde, Secretaria de Atenção à Saúde, Departamento de Ações Programáticas Estratégicas. [livro online]. Brasília; 2014.

5 - Sankar C; Mundkur N. Cerebral palsy: definition, classification, etiology and early diagnosis. Indian J Pediatr. 2005 [citado Out 2019]. Acesso em: https://www.ncbi.nlm.nih. 
gov/pubmed/16272660

6 - Lacoste M, Therrien M, Prince F. Stability of children with cerebral palsy in their wheelchair seating: Perceptions of parents and therapists. Disability \& Rehabilitation: Assistive Technology. 2009 [citado Out 2019]. Acesso em: https:// www.researchgate.net/publication/24309116_ Stability_of_children_with_cerebral_palsy_in_their_wheelchair_seating_Perceptions_of_ parents_and_therapists

7-Silva AS, Valenciano PJ, Fujisawa DS. Atividade Lúdica na Fisioterapia em Pediatria: Revisão de Literatura. Rev. bras. educ. espec. 2017 [citado Out 2019]. Acesso em: http://www.scielo.br/ pdf/rbee/v23n4/1413-6538-rbee-23-04-0623. pdf

8 - Sari FL, Marcon SS. Participação da Família no Trabalho Fisioterapêutico em Crianças com Paralisia Cerebral. Ver Bras Crescimento Desenvolvimento Hum. 2008 [citado Out 2019]. Acesso em: https://pdfs.semanticscholar.org/8 89c/72b8c45ca8ca3509de9c681697182e19a577. pdf

9 - Capucho PY, Carnier SADC, Souza P, Castro DC, Finocchio AP, Oliveira DM, et al. Paralisia Cerebral - Membros Inferiores: Reabilitação. Revistas Usp. 2012 [citado Out 2019]. Acesso em: https://www.revistas.usp.br/actafisiatrica/ article/view/103693/102162

10 - Houwink A, Aarts PBM, Geurts ACH, Steenbergen B. A neurocognitive perspective on developmental disregard in children with hemiplegic cerebral palsy. Research in Developmental Disabilities. 2011 [citado Out 2019]. Acesso em: https://www.academia. edu/6155511/A_neurocognitive_perspective_ on_developmental_disregard_in_children_with_hemiplegic_cerebral_palsy

11 - Souza T. Conhecer e interagir: transtorno de déficit de atenção e hiperatividade e transtorno do espectro autista. João Pessoa: Universidade Federal da Paraíba, 2015 [citado Out 2019]. Acesso em: https://repositorio.ufpb.br/jspui/ bitstream/123456789/1211/1/TAS19092016. pdf

12 - Graham HK, Rosenbaum P, Dan B, Lin JP, Damiano DL, Becher JG, et al. Cerebral palsy. Nat Rev Dis Primers. 2016 [citado Out 2019]. Acesso em: https://www.ncbi.nlm.nih.gov/ pubmed/27188686

13 - Davids JR, Bagley AM. Identification of Common Gait Disruption Patterns in Children With Cerebral Palsy. J Am Acad Orthop Surg. 2014 [citado Out 2019]. Acesso em: https: P/ docksci.com/identification-of-common-gaitdisruption-patterns-in-children-with-cerebralpals_5a7e9dded64ab20d82d0d0c3.html

14 - Unger M, Jelsma J, Stark C. Effect of a trunktargeted intervention using vibration on posture and gait in children with spastic type cerebral palsy: a randomized control trial. Developmental Neurorehabilitation. 2013 [citado Out 2019]. Acesso em: https://www.researchgate.net/ publication/235904455_Effect_of_a_trunktargeted_intervention_using_vibration_on_ posture_and_gait_in_children_with_spastic_type_cerebral_palsy_A_randomized_control_trial

15 - Heyrman L, Desloovere K, Molenaers G, Verheyden G, Klingels K, Monbaliu E. Clinical characteristics of impaired trunk control in children with spastic cerebral palsy. Res Dev Disabil. 2013 [citado Out 2019]. Acesso em: https://www.ncbi.nlm.nih.gov/ pubmed/23000634

16 - Mancini MC, Alves ACM, Schaper C, Figueiredo EM, Sampaio RF, Coelho ZAC, et al. Gravidade da paralisia cerebral e desempenho funcional. Ver. Bras. Fisiotera, 2004 [citado Out 2019]. Acesso em: http://bases.bireme.br/cgi$\mathrm{bin} / \mathrm{wxislind}$.exe/iah/online/?IsisScript=iah/ iah. $x i s \& s r c=$ google\&base $=$ LILACS\&lang $=p \& n$ extAction $=\operatorname{lnk} \&$ exprSearch $=404403 \&$ indexSear $\mathrm{ch}=\mathrm{ID}$

OBSERVAÇÃO: Os autores declaram não existir conflitos de interesse de qualquer natureza. 